\title{
ON THE CONVERGENCE AND SUMMABILITY OF SERIES WITH RESPECT TO BLOCK-ORTHONORMAL SYSTEMS
}

\author{
G. NADIBAIDZE
}

\begin{abstract}
Statements connected with the so-called block-orthonormalized systems are given. The convergence and summability almost everywhere by the $(c, 1)$ method with respect to such systems are considered. In particular, the well-known theorems of MenshovRademacher and Kacmarz on the convergence and (c,1)-summability almost everywhere of orthogonal series are generalized.
\end{abstract}

1. The so-called block-orthonormal systems were introduced by V. F. Gaposhkin who obtained the first results [1] for series with respect to such systems. In particular, he generalized the well-known MenshovRademacher theorem. This paper presents the results on the convergence and $(c, 1)$-summability almost everywhere of series with respect to blockorthonormal systems. These results were announced in [2] and [3] but here some of them are formulated in a slightly different form.

Let $\left\{N_{k}\right\}$ be a strictly increasing sequence of natural numbers and $\Delta_{k}=$ $\left(N_{k}, N_{k+1}\right], k=1,2, \ldots$.

Definition $1([1])$. Let $\left\{\varphi_{n}\right\}$ be a system of functions from $L^{2}(0,1)$. $\left\{\varphi_{n}\right\}$ will be called a $\Delta_{k}$-orthonormal system $\left(\Delta_{k}\right.$-ONS) if:

(1) $\left\|\varphi_{n}\right\|_{2}=1, \quad n=1,2, \ldots$;

(2) $\left(\varphi_{i}, \varphi_{j}\right)=0$ for $i, j \in \Delta_{k}, i \neq j, k \geq 1$.

Definition 2. A positive nondecreasing sequence $\{\omega(n)\}$ will be called the Weyl multiplier for the convergence $((c, 1)$-summability) a.e. of series with respect to the $\Delta_{k}$-ONS $\left\{\varphi_{n}(x)\right\}$ if the convergence of the series

$$
\sum_{n=1}^{\infty} a_{n}^{2} \omega(n)
$$

1991 Mathematics Subject Classification. 42C20.

Key words and phrases. Block-orthonormal systems, Weyl multiplier, convergence and $(\mathrm{c}, 1)$-summability almost everywhere of block-normal systems.

$$
517
$$

1072-947X/95/0900-0517\$7.50/0 (C) 1995 Plenum Publishing Corporation 
guarantees the convergence $((c, 1)$-summability) a.e. of the series

$$
\sum_{n=1}^{\infty} a_{n} \varphi(x)
$$

2. Let the sequence $\left\{N_{k}\right\}$ be fixed and $\Delta_{k}=\left(N_{k}, N_{k+1}\right]$. Without loss of generality it can be assumed that

$$
N_{0}=0, \quad N_{1}=1, \quad \omega(0)=1 .
$$

We have

Theorem 1. In order that a positive nondecreasing sequence $\{\omega(n)\}$ be the Weyl multiplier for the convergence a.e. of series with respect to any $\Delta_{k}$-ONS, it is necessary and sufficient that the following two conditions be fulfilled:

(a) $\sum_{k=1}^{\infty} \frac{1}{\omega\left(N_{k}\right)}<\infty$;

(b) $\log _{2}^{2} n=O(\omega(n))$ for $n \rightarrow \infty$.

Proof. Sufficiency. Let the conditions of the theorem be fulfilled and for the sequence $\left\{a_{n}\right\}$

$$
\sum_{n=1}^{\infty} a_{n}^{2} \omega(n)<\infty .
$$

We introduce

$$
\psi_{k}(x)=\sum_{n=N_{k}+1}^{N_{k+1}} a_{n} \varphi_{n}(x), \quad k=0,1,2, \ldots .
$$

Then

$$
\begin{gathered}
\sum_{k=0}^{\infty}\left\|\psi_{k}(x)\right\|_{1} \leq \sum_{k=0}^{\infty}\left\|\psi_{k}(x)\right\|_{2}=\sum_{k=0}^{\infty}\left\|\psi_{k}(x)\right\|_{2}\left(\omega\left(N_{k}\right)\right)^{\frac{1}{2}}\left(\omega\left(N_{k}\right)\right)^{-\frac{1}{2}} \leq \\
\leq \sum_{k=0}^{\infty}\left\|\psi_{k}(x)\right\|_{2}^{2} \omega\left(N_{k}\right) \sum_{k=0}^{\infty} \frac{1}{\omega\left(N_{k}\right)}=\sum_{k=0}^{\infty}\left(\sum_{n=N_{k}+1}^{N_{k+1}} a_{n}^{2}\right) \omega\left(N_{k}\right) \sum_{k=0}^{\infty} \frac{1}{\omega\left(N_{k}\right)} \leq \\
\leq \sum_{n=1}^{\infty} a_{n}^{2} \omega(n) \sum_{k=0}^{\infty} \frac{1}{\omega\left(N_{k}\right)}<\infty,
\end{gathered}
$$

which by the Levy theorem implies that

$$
\sum_{k=0}^{\infty}\left|\psi_{k}(x)\right|<\infty \quad \text { a.e. }
$$


Therefore the sequence $S_{N_{k}}(x)$, where

$$
S_{k}(x)=\sum_{n=1}^{k} a_{n} \varphi_{n}(x)
$$

converges a.e.

Let

$$
\delta_{k}(x)=\max _{N_{k}<j \leq N_{k+1}}\left|\sum_{n=N_{k}+1}^{j} a_{n} \varphi(x)\right|, \quad k \geq 1 .
$$

Using the Kantorovich inequality, we obtain

$$
\left\|\delta_{k}(x)\right\|_{2}^{2} \leq c \sum_{n=N_{k}+1}^{N_{k+1}} a_{n}^{2} \log _{2}^{2} n, \quad k \geq 1 .
$$

Now

$$
\sum_{k=0}^{\infty}\left\|\delta_{k}(x)\right\|_{2}^{2} \leq c \sum_{k=0}^{\infty} \sum_{n=N_{k}+1}^{N_{k+1}} a_{n}^{2} \log _{2}^{2} n \leq c \sum_{n=1}^{\infty} a_{n}^{2} \omega(n)<\infty, 1
$$

from which it follows that $\lim _{k \rightarrow \infty} \delta_{k}(x)=0$ for a.e. $x \in(0,1)$. This together with the proven convergence almost everywhere of the series $S_{N_{k}}(x)$ guarantees the convergence of series (1) a.e. on $(0,1)$.

Necessity.

(1) Let

$$
\sum_{k=1}^{\infty} \frac{1}{\omega\left(N_{k}\right)}=\infty
$$

Then there exist numbers $c_{k}>0$ such that

$$
\sum_{k=1}^{\infty} c_{k}^{2} \omega\left(N_{k}\right)<\infty \text { and } \sum_{k=1}^{\infty} c_{k}=\infty
$$

Let $\Phi_{N_{k}}(x)=1(k=1,2, \ldots ; x \in(0,1))$ and choose as other functions $\Phi_{n}(x)\left(n \in N, n \neq N_{k}, k=1,2, \ldots\right)$ an arbitrary ONS orthogonal to 1. The system $\left\{\Phi_{n}(x)\right\}$ is an $\Delta_{k}$-ONS. Take $b_{n}=0\left(n \neq N_{1}, N_{2}, \ldots\right), b_{N_{k}}=c_{k}$ $(k=1,2, \ldots)$. Then

$$
\sum_{n=1}^{\infty} b_{n} \Phi_{n}(x)=\sum_{k=1}^{\infty} c_{k}=\infty, \quad x \in(0,1),
$$

though

$$
\sum_{n=1}^{\infty} b_{n} \omega(n)=\sum_{k=1}^{\infty} c_{k}^{2} \omega\left(N_{k}\right)<\infty
$$

\footnotetext{
${ }^{1}$ In what follows $c$ will denote, generally speaking, various absolute constants.
} 
The necessity of condition (1) is proved.

(2) If equality (b) is not fulfilled, then

$$
\frac{\log _{2}^{2} 2^{k}}{\omega\left(2^{k}\right)} \geq \frac{1}{4} \frac{\log _{2}^{2} 2^{k+1}}{\omega\left(2^{k}\right)} \geq \frac{1}{4} \frac{\log _{2}^{2} n}{\omega(n)}, \quad n \in\left(2^{k}, 2^{k+1}\right] \quad k=1,2, \ldots,
$$

which implies that the equality

$$
\log _{2}^{2} 2^{k}=O\left(\omega\left(2^{k}\right)\right) \text { for } k \rightarrow \infty
$$

is not fulfilled either. Therefore we can find an increasing sequence of natural numbers $q_{j}, j=1,2, \ldots$, such that

$$
1 \leq \sqrt{\omega\left(2^{q_{j}+1}\right)}<\frac{q_{j}}{j^{3}}, \quad j=1,2, \ldots .
$$

Inequality (2) makes it possible to construct an orthonormal system $\left\{\Phi_{n}(x)\right\}$ (which simultaneously will also be a $\Delta_{k}$-ONS) and a sequence $\left\{b_{n}\right\}$ (see [4], p. 298, the proof of Menshov's theorem) such that

$$
\sum_{n=1}^{\infty} b_{n}^{2} \omega(n)<\infty
$$

but the series

$$
\sum_{n=1}^{\infty} b_{n} \Phi_{n}(x)
$$

diverges a.e. on $(0,1)$.

Remark 1. The application of the proven theorem to orthonormal systems allows us to formulate the Menshov-Rademacher theorem as follows:

In order that a positive nondecreasing sequence $\{\omega(n)\}$ be the Weyl multiplier for the convergence a.e. of series with respect to any orthonormal systems, it is necessary and sufficient that the equality

$$
\log _{2}^{2} n=O(\omega(n)) \quad \text { as } n \rightarrow \infty
$$

be fulfilled.

Remark 2. If

$$
\omega(n)=\log _{2}^{2} n,
$$

then condition (b) of Theorem 1 is fulfilled and we obtain Gaposhkin's theorem [1, Proposition 1]. 
Remark 3. If

$$
N_{k}=\left[2^{k^{\alpha}}\right], \quad 0<\alpha \leq \frac{1}{2}, 1
$$

then $\log _{2}^{2} n$ will be the Weyl multiplier for the convergence a.e. not for each $\Delta_{k}$-ONS. From Theorem 1 it follows that in that case

$$
\omega(n)=\log _{2}^{\frac{1}{\alpha}+\varepsilon} n, \quad \varepsilon>0,
$$

is the Weyl multiplier.

Analogously, if

$$
N_{k}=\left[k^{\alpha}\right], \quad \alpha \geq 1
$$

then

$$
\omega(n)=n^{\frac{1}{\alpha}} \log _{2}^{1+\varepsilon} n, \quad \varepsilon>0 .
$$

Also note that in both cases one should not take $\varepsilon=0$.

3. Here a necessary and sufficient condition is established to be imposed on the sequence $\left\{N_{k}\right\}$ so that the well-known Kacmarz theorem on the $(c, 1)$ summability a.e. of series with respect to orthonormal systems (see [5], p. 223 , theorem [5.8.6]) remains valid also with respect to block-orthonormal systems. Moreover, a generalization of the Kacmarz theorem is given for a $\Delta_{k}$-ONS.

In what follows we shall use the notation

$$
\sigma_{n}(x)=\frac{1}{n} \sum_{i=1}^{n} S_{i}(x), \quad k(n)=\max \left\{k: N_{k}<n\right\} .
$$

Lemma 1. Let the sequence $\left\{N_{k}\right\}$ be fixed, $\left\{\varphi_{n}\right\}$ be an arbitrary $\Delta_{k^{-}}$ ONS and for a positive nondecreasing sequence $\{\omega(n)\}$ let there be given

$$
\min \left\{k: N_{k} \geq n\right\}+n^{2} \sum_{k: N_{k} \geq n} \frac{1}{N_{k}^{2}}=O(\omega(n)) \text { for } n \rightarrow \infty .
$$

Then the condition

$$
\sum_{n=1}^{\infty} a_{n}^{2} \omega(n)<\infty
$$

implies the convergence a.e. of the series

$$
\sum_{n=2}^{\infty} n\left(\sigma_{n}(x)-\sigma_{n-1}(x)\right)^{2}
$$

\footnotetext{
${ }^{1}[p]$ denotes the integer part of the number $p$.
} 
Proof. Let conditions (3) and (4) be fulfilled. Then

$$
\begin{gathered}
\int_{0}^{1} n\left(\sigma_{n}(x)-\sigma_{n-1}(x)\right)^{2} d x=\frac{1}{n(n-1)^{2}} \int_{0}^{1}\left(\sum_{i=1}^{n} a_{i}(i-1) \varphi_{i}(x)\right)^{2} d x \leq \\
\leq \frac{4}{n^{3}} \int_{0}^{1}\left(\sum_{i=1}^{N_{k(n)}} a_{i}(i-1) \varphi_{i}(x)+\sum_{i=N_{k(n)}+1}^{n} a_{i}(i-1) \varphi(x)\right)^{2} d x \leq \\
\leq \frac{8}{n^{3}}\left[\int_{0}^{1}\left(\sum_{j=0}^{k(n)-1} \sum_{i=N_{j}+1}^{N_{j+1}} a_{i}(i-1) \varphi_{i}(x)\right)^{2} d x+\right. \\
\leq \frac{8}{n^{3}}\left[\begin{array}{c}
\left.\left.\int_{0}^{1}(n) \sum_{i=N_{k(n)}+1}^{n} a_{i}(i-1) \varphi_{i}(x)\right)^{2}\right] \leq \\
k(n)-1
\end{array} \int_{0}^{1}\left(\sum_{i=N_{j}+1}^{N_{j+1}} a_{i}(i-1) \varphi_{i}(x)\right)^{2} d x+\sum_{i=N_{k(n)}+1}^{n} a_{i}^{2}(i-1)^{2}\right]= \\
=\frac{8}{n^{3}}\left[k(n) \sum_{i=1}^{N_{k(n)}} a_{i}^{2}(i-1)^{2}+\sum_{i=N_{k(n)}+1}^{n} a_{i}^{2}(i-1)^{2}\right] \leq \\
\leq \frac{8}{n^{3}}\left[k(n) \sum_{i=1}^{N_{k(n)}} a_{i}^{2} i^{2}+\sum_{i=N_{k(n)}+1}^{n} a_{i}^{2} i^{2}\right], \quad n \geq 2 .
\end{gathered}
$$

Therefore

$$
\begin{gathered}
\sum_{n=2}^{\infty} \int_{0}^{1} n\left(\sigma_{n}(x)-\sigma_{n-1}(x)\right)^{2} d x \leq 8 \sum_{k=0}^{\infty} \sum_{n=N_{k}+1}^{N_{k+1}} \frac{1}{n^{3}}\left(k(n) \sum_{i=1}^{N_{k(n)}} a_{i}^{2} i^{2}+\right. \\
\left.+\sum_{i=N_{k(n)}+1}^{n} a_{i}^{2} i^{2}\right)=8 \sum_{k=0}^{\infty}\left(\sum_{n=N_{k}+1}^{N_{k+1}} \sum_{i=1}^{N_{k}} \frac{k}{n^{3}} a_{i}^{2} i^{2}+\sum_{n=N_{k}+1}^{N_{k+1}} \sum_{i=N_{k}+1}^{n} \frac{1}{n^{3}} a_{i}^{2} i^{2}\right)= \\
=8 \sum_{i=1}^{\infty} a_{i}^{2} i^{2} \sum_{k: N_{k} \geq i} k \sum_{n=N_{k}+1}^{N_{k+1}} \frac{1}{n^{3}}+8 \sum_{k=0}^{\infty} \sum_{i=N_{k}+1}^{N_{k+1}} a_{i}^{2} i^{2} \sum_{n=i}^{N_{k+1}} \frac{1}{n^{3}} \leq \\
\leq 8 \sum_{i=1}^{\infty} a_{i}^{2} i^{2} \sum_{k=k(i)+1} k\left(\frac{1}{N_{k}^{2}}-\frac{1}{N_{k+1}^{2}}\right)+c \sum_{k=0}^{\infty} \sum_{i=N_{k}+1}^{N_{k+1}} a_{i}^{2}=
\end{gathered}
$$




$$
\begin{gathered}
\quad=8 \sum_{i=1}^{\infty} a_{i}^{2} i^{2}\left[(k(i)+1) \frac{1}{N_{k(i)+1}^{2}}+\sum_{k=k(i)+2}^{\infty} \frac{1}{N_{k}^{2}}\right]+c \sum_{i=1}^{\infty} a_{i}^{2}< \\
<c \sum_{i=1}^{\infty} a_{i}^{2}\left(\min \left\{k: N_{k} \geq i\right\}+i^{2} \sum_{k: N_{k} \geq i} \frac{1}{N_{k}^{2}}\right) \leq c \sum_{i=1}^{\infty} a_{i}^{2} \omega(i)<\infty,
\end{gathered}
$$

from which by the Levy theorem we obtain

$$
\sum_{n=2}^{\infty} n\left(\sigma_{n}(x)-\sigma_{n-1}(x)\right)^{2}<\infty \quad \text { a.e. }
$$

Lemma 2. Let $\left\{N_{k}\right\}$ be a given sequence, $\left\{\varphi_{n}(x)\right\}$ be an arbitrary $\Delta_{k^{-}}$ ONS, and conditions (3), (4) be fulfilled. Then for the corresponding series (1) the convegence a.e. of the sequence $\left\{S_{2^{n}}(x)\right\}$ is equivalent to the convergence a.e. of the sequence $\left\{\sigma_{2^{n}}(x)\right\}$.

Proof. Let conditions (3) and (4) be fulfilled. We have

$$
S_{n}(x)-\sigma_{n}(x)=\frac{1}{n} \sum_{i=1}^{n} a_{i}(i-1) \varphi_{i}(x) .
$$

Then

$$
\begin{aligned}
& \int_{0}^{1}\left(S_{2^{n}}(x)-\sigma_{2^{n}}(x)\right)^{2} d x=\int_{0}^{1} \frac{1}{4^{n}}\left(\sum_{i=1}^{N_{k\left(2^{n}\right)}} a_{i}(i-1) \varphi_{i}(x)+\right. \\
+ & \left.\sum_{i=N_{k\left(2^{n}\right)+1}}^{2^{n}} a_{i}(i-1) \varphi_{i}(x)\right) d x \leq \frac{2}{4^{n}}\left[k\left(2^{n}\right) \sum_{i=1}^{N_{k\left(2^{n}\right)}} a_{i}^{2}(i-1)^{2}+\right. \\
+ & \left.\sum_{i=N_{k\left(2^{n}\right)}+1}^{2^{n}} a_{i}^{2}(i-1)^{2}\right] \leq \frac{2}{4^{n}}\left[k\left(2^{n}\right) \sum_{i=1}^{N_{k\left(2^{n}\right)}} a_{i}^{2} i^{2}+\sum_{i=N_{k\left(2^{n}\right)}+1}^{2^{n}} a_{i}^{2} i^{2}\right] .
\end{aligned}
$$

Therefore

$$
\begin{gathered}
\sum_{n=1}^{\infty} \int_{0}^{1}\left(S_{2^{n}}(x)-\sigma_{2^{n}}(x)\right)^{2} d x \leq 2\left(\sum_{n=1}^{\infty} \frac{k\left(2^{n}\right)}{4^{n}} \sum_{i=1}^{N_{k\left(2^{n}\right)}} a_{i}^{2} i^{2}+\right. \\
\left.+\sum_{n=1}^{\infty} \frac{1}{4^{n}} \sum_{i=N_{k\left(2^{n}\right)}+1}^{2^{n}} a_{i}^{2} i^{2}\right)=2\left(J_{1}+J_{2}\right) .
\end{gathered}
$$


We have

$$
\begin{gathered}
J_{1}=\sum_{n=1}^{\infty} \frac{k\left(2^{n}\right)}{4^{n}} \sum_{i=1}^{N_{k\left(2^{n}\right)}} a_{i}^{2} i^{2}=\sum_{k=1}^{\infty} \sum_{\log _{2}} \sum_{N_{k}<n \leq \log _{2} N_{k+1}} \frac{k\left(2^{n}\right)}{4^{n}} \sum_{i=1}^{N_{k\left(2^{n}\right)}} a_{i}^{2} i^{2}= \\
=\sum_{k=1}^{\infty} \sum_{\log _{2} N_{k}<n \leq \log _{2} N_{k+1}} \frac{k}{4^{n}} \sum_{i=1}^{N_{k}} a_{i}^{2} i^{2}= \\
=\sum_{k=1}^{\infty}\left(\sum_{\log _{2} N_{k}<n \leq \log _{2} N_{k+1}} \frac{k}{4^{n}}\right) \sum_{i=1}^{N_{k}} a_{i}^{2} i^{2}= \\
=\sum_{i=1}^{\infty} a_{i}^{2} i^{2} \sum_{k=k(i)+1}^{\infty}\left(\sum_{\log _{2}} \sum_{k=1}^{\infty} a_{i}^{2} i^{2}\left[(k(i)+1) \sum_{n>\log _{2} N_{2} N_{k+1}} \frac{k}{4^{n}}\right)=\right. \\
\leq \sum_{i=1}^{\infty} a_{i}^{2} i^{2}\left[(k(i)+1) \frac{1}{4^{n}}+\sum_{k=k(i)+2}^{\infty} \frac{1}{N_{k(i)+1}^{2}}+\frac{4}{3} \sum_{k=k(i)+2}^{\infty} \frac{1}{N_{k}^{2}}\right] \leq \\
\leq \frac{4}{3} \sum_{i=1}^{\infty} a_{i}^{2} i^{2}\left[\frac{1}{i^{2}} \min \left\{k: N_{k} \geq i\right\}+\sum_{k: N_{k} \geq i} \frac{1}{4_{k}^{2}}\right] \leq c \sum_{i=1}^{\infty} a_{i}^{2} \omega(i)<\infty
\end{gathered}
$$

and

$$
\begin{gathered}
J_{2}=\sum_{n=1}^{\infty} \frac{1}{4^{n}} \sum_{i=N_{k\left(2^{n}\right)}+1} a_{i}^{2} i^{2} \leq \sum_{n=1}^{\infty} \frac{1}{4^{n}} \sum_{i=1}^{2^{n}} a_{i}^{2} i^{2}= \\
=\sum_{i=1}^{\infty} a_{i}^{2} i^{2} \sum_{2^{n} \geq i} \frac{1}{4^{n}} \leq c \sum_{i=1}^{\infty} a_{i}^{2}<\infty .
\end{gathered}
$$

Therefore

$$
\sum_{n=1}^{\infty} \int_{0}^{1}\left(S_{2^{n}}(x)-\sigma_{2^{n}}(x)\right)^{2}<\infty
$$

from which it follows that

$$
\sum_{n=1}^{\infty} \int_{0}^{1}\left(S_{2^{n}}(x)-\sigma_{2^{n}}(x)\right)^{2}<\infty \quad \text { a.e. }
$$

and therefore

$$
\lim _{n \rightarrow \infty} \int_{0}^{1}\left(S_{2^{n}}(x)-\sigma_{2^{n}}(x)\right)^{2}=0 \quad \text { a.e. }
$$


Theorem 2. Let $\left\{N_{k}\right\}$ be a given sequence, $\left\{\varphi_{n}(x)\right\}$ be an arbitrary $\Delta_{k}$ ONS, and conditions (3), (4) be fulfilled. Then for series (1) to be $(c, 1)-$ convergent a.e. it is necessary and sufficient that the subsequence of partial sums $\left\{S_{2^{n}}(x)\right\}$ of (1) be convergent a.e.

Proof. Sufficiency. Let conditions (3), (4) be fulfilled and the subsequence $\left\{S_{2^{n}}(x)\right\}$ of the corresponding series (1) converge a.e. Then by Lemma 3 the subsequence $\left\{\sigma_{2^{n}}(x)\right\}$ also converges a.e. and we have

$$
\begin{gathered}
\sup _{k \in\left(2^{n}, 2^{n+1}\right]}\left(\sigma_{k}(x)-\sigma_{2^{n}}(x)\right)^{2}=\left(\sup _{k \in\left(2^{n}, 2^{n+1}\right]} \sum_{i=2^{n}+1}^{k}\left(\sigma_{i}(x)-\sigma_{i-1}(x)\right)\right)^{2} \leq \\
\leq \sum_{i=2^{n}+1} i\left(\sigma_{i}(x)-\sigma_{i-1}(x)\right)^{2},
\end{gathered}
$$

which by Lemma 1 implies that $\left\{\sigma_{n}(x)\right\}$ converges a.e., i.e., series (1) is $(c, 1)$-summable a.e.

Necessity. Let conditions (3), (4) be fulfilled and series (1) be $(c, 1)$ summable a.e. Then $\left\{\sigma_{2^{n}}(x)\right\}$ converges almost everywhere and by Lemma $2\left\{S_{2^{n}}(x)\right\}$, too, converges almost everywhere.

Lemma 3. If

$$
\sum_{k=3}^{\infty} \frac{1}{\left(\log _{2} \log _{2} N_{k}\right)^{2}}<\infty
$$

then

$$
\min \left\{k: N_{k} \geq n\right\}+n^{2} \sum_{k: N_{k} \geq n} \frac{1}{N_{k}^{2}}=O\left(\left(\log _{2} \log _{2} n\right)^{2}\right) \quad \text { for } n \rightarrow \infty .
$$

Proof. Let

$$
\sum_{k=2}^{\infty} \frac{1}{\left(\log _{2} \log _{2} N_{k}\right)^{2}}<\infty
$$

Then

$$
\lim _{k \rightarrow \infty} \frac{k}{\left(\log _{2} \log _{2} N_{k}\right)^{2}}=0
$$

and therefore for sufficiently large $k$ 's we have

$$
2^{2^{\sqrt{k}}}<N_{k}
$$

By definition, $n \in\left(N_{k(n)}, N_{k(n)+1}\right]$. Putting

$$
q(n)= \begin{cases}k(n)+1, & \text { if } 2^{2^{\sqrt{k(n)+1}}} \geq n, \\ m, & \text { if } 2^{2^{\sqrt{k(n)+1}}}<n \text { and } 2^{2^{\sqrt{m-1}}} \leq n<2^{2^{\sqrt{m}}},\end{cases}
$$


for sufficiently large $n$ 's we have

$$
\begin{aligned}
& \sum_{k: N_{k} \geq n} \frac{1}{N_{k}^{2}}=\sum_{k=k(n)+1} \frac{1}{N_{k}^{2}}=\sum_{k=k(n)+1}^{q(n)-1} \frac{1}{N_{k}^{2}}+\sum_{k=q(n)}^{\infty} \frac{1}{N_{k}^{2}} \leq \\
& \leq \frac{q(n)-k(n)-1}{N_{k(n)+1}}+\sum_{k=q(n)}^{\infty} \frac{1}{\left(2^{2^{\sqrt{k}}}\right)^{2}} \leq \frac{q(n)-k(n)-1}{n^{2}}+ \\
& +\frac{c}{\left(2^{2 \sqrt{q(n)}}\right)^{2}} \leq \frac{q(n)-k(n)-1}{n^{2}}+\frac{c}{n^{2}} \leq c \frac{\left(\log _{2} \log _{2} n\right)^{2}}{n^{2}} .
\end{aligned}
$$

Therefore for sufficiently large $n$ 's

$$
\begin{aligned}
\min \left\{k: N_{k} \geq n\right\}+n^{2} & \sum_{k: N_{k} \geq n} \frac{1}{N_{k}^{2}} \leq k(n)+1+n^{2} c \frac{\left(\log _{2} \log _{2} n\right)^{2}}{n^{2}} \leq \\
\leq & c\left(\log _{2} \log _{2} n\right)^{2} .
\end{aligned}
$$

Theorem 3. Let the sequence $\left\{N_{k}\right\}$ be fixed. In order that the condition

$$
\sum_{n=2}^{\infty} a_{n}^{2}\left(\log _{2} \log _{2} n\right)^{2}<\infty
$$

guarantee the convergence a.e. of the sequence $\left\{S_{2^{k}}(x)\right\}$ for series (1) with respect to any $\Delta_{k}$-ONS $\left\{\varphi_{n}(x)\right\}$, it is necessary and sufficient that the condition

$$
\sum_{k=3}^{\infty} \frac{1}{\left(\log _{2} \log _{2} N_{k}\right)^{2}}<\infty
$$

be fulfilled.

Proof. Sufficiency. Let conditions (5) and (6) be fulfilled. Define the sequence of natural numbers $\left\{M_{i}\right\}$ by the recurrent formula

$$
\begin{aligned}
M_{1}= & N_{1}=1, \\
M_{i}= & \min \left\{\min \left\{N_{k}: M_{k}>M_{i-1}, k \in N\right\},\right. \\
& \left.\min \left\{2^{m}: 2^{m}>M_{i-1}, m \in N\right\}\right\}, \quad i \geq 2,
\end{aligned}
$$

i.e., $\left\{M_{i}\right\}$ is the increasing sequence whose terms have the form $N_{k}$ or $2^{m}$, $k \geq 1, m \geq 1$.

Assume that $N_{i}=M_{k_{i}}, i \geq 1$, and $k_{0}=0$. Clearly,

$$
M_{i}<2^{i}, \quad i \geq 1,
$$


and

$$
\log _{2} M_{p}+i+1 \geq p \text { for } p \in\left(k_{i}, k_{i+1}\right], \quad i \geq 0 .
$$

Now, applying condition (6) and inequality (9), for sufficiently large $i$ 's and $p \in\left(k_{i}, k_{i+1}\right]$ we have

$$
\begin{gathered}
p \leq \log _{2} M_{p}+i+1 \leq \log _{2} M_{p}+\log _{2} 2^{2^{\sqrt{i}}} \leq \log _{2} M_{p}+\log _{2} N_{i}= \\
=\log _{2} M_{p}+\log _{2} M_{k_{i}} \leq 2 \log _{2} M_{p} .
\end{gathered}
$$

Set

$$
b_{n}=\left(\sum_{j=M_{n}+1}^{M_{n+1}} a_{j}^{2}\right)^{\frac{1}{2}}, \quad \psi_{n}(x)= \begin{cases}\frac{1}{b_{n}} \sum_{j=M_{n}+1}^{M_{n+1}} a_{j} \varphi_{j}(x), & \text { for } b_{n} \neq 0, \quad n \geq 1 . \\ \varphi_{M_{n}+1}(x), & \text { for } b_{n}=0,\end{cases}
$$

Clearly, $\left\{\psi_{n}(x)\right\}$ is a $\left(k_{i}, k_{i+1}\right]$-ONS. Moreover, by condition (6) and inequality (8) we have

$$
\sum_{i=3}^{\infty} \frac{1}{\log _{2}^{2} k_{i}} \leq \sum_{i=3}^{\infty} \frac{1}{\left(\log _{2} \log _{2} M_{k_{i}}\right)^{2}}=\sum_{i=3}^{\infty} \frac{1}{\left(\log _{2} \log _{2} N_{i}\right)^{2}}<\infty
$$

and by (5) and (10)

$$
\begin{gathered}
\sum_{n=1}^{\infty} b_{n}^{2} \log _{2}^{2} n=\sum_{n=1}\left(\sum_{j=M_{n}+1}^{M_{n+1}} a_{j}^{2}\right) \log _{2}^{2} n \leq c \sum_{n=1}^{\infty}\left(\sum_{j=M_{n}+1}^{M_{n+1}} a_{j}^{2}\right) \times \\
\times\left(\log _{2} \log _{2} M_{n}\right)^{2} \leq c \sum_{n=1}^{\infty} \sum_{j=M_{n}+1}^{M_{n+1}} a_{j}^{2}\left(\log _{2} \log _{2} j\right)^{2}<\infty
\end{gathered}
$$

Thus the conditions of V. Gaposhkin's theorem (see [1], Proposition 1) are fulfilled for $\left(k_{i}, k_{i+1}\right]$-ONS $\left\{\psi_{n}(x)\right\}$ and the sequence $\left\{b_{n}\right\}$. Therefore the series

$$
\sum_{n=1}^{\infty} b_{n} \psi_{n}(x)
$$

converges almost everywhere, which, in particular, guarantees the convergence a.e. of the sequence $\left\{S_{2^{k}}(x)\right\}$ for the corresponding series (1).

Necessity. Let

$$
\sum_{k=3}^{\infty} \frac{1}{\left(\log _{2} \log _{2} N_{k}\right)^{2}}=\infty
$$

Then there exist numbers $c_{k}>0$ such that

$$
\sum_{k=2}^{\infty} c_{k}^{2}\left(\log _{2} \log _{2} N_{k}\right)^{2}<\infty, \quad \sum_{k=1}^{\infty} c_{k}=\infty
$$


Take $\Phi_{N_{k}}(x) \equiv 1(k \geq 1)$ and as other functions $\Phi_{n}(x)\left(n \neq N_{1}, N_{2}, \ldots\right)$ choose an arbitrary ONS orthogonal to 1 . The system $\left\{\Phi_{n}(x)\right\}$ is a $\Delta_{k}$-ONS. Let $b_{N_{k}}=c_{k}(k \geq 1)$ and $b_{n}=0\left(n \neq N_{1}, N_{2}, \ldots\right)$. Then

$$
\sum_{n=2}^{\infty} b_{n}^{2}\left(\log _{2} \log _{2} n\right)^{2}=\sum_{k=2}^{\infty} c_{k}^{2}\left(\log _{2} \log _{2} N_{k}\right)^{2}<\infty,
$$

but

$$
\sum_{n=1}^{\infty} b_{n} \Phi_{n}(x)=\sum_{k=1}^{\infty} b_{N_{k}}=\sum_{k=1}^{\infty} c_{k}=\infty, \quad x \in(0,1)
$$

i.e., for the series

$$
\sum_{n=1}^{\infty} b_{n} \Phi_{n}(x)
$$

the sequence $\left\{S_{2^{k}}(x)\right\}$ diverges everywhere.

Theorem 4. Let the sequence $\left\{N_{k}\right\}$ be fixed. In order that the sequence $\left\{\left(\log _{2} \log _{2} n\right)^{2}\right\}$ be the Weyl multiplier for the $(c, 1)$-summability a.e. of series with respect to any $\Delta_{k}$-ONS, it is necessary and sufficient that condition (6) be fulfilled.

Proof. Sufficiency. Let conditions (5) and (6) be fulfilled. Then by Theorem 3 the sequence $\left\{S_{2^{k}}(x)\right\}$ converges a.e. for series (1), while by Lemma 3

$$
\min \left\{k: N_{k} \geq n\right\}+n^{2} \sum_{k: N_{k} \geq n} \frac{1}{N_{k}^{2}}=O\left(\left(\log _{2} \log _{2} n\right)^{2}\right), \quad n \rightarrow \infty,
$$

holds and therefore series $(1)$ is $(c, 1)$-summable by Theorem 2 .

Necessity. Let

$$
\sum_{k=3}^{\infty} \frac{1}{\left(\log _{2} \log _{2} N_{k}\right)^{2}}=\infty
$$

Construct the $\Delta_{k}$-ONS $\left\{\Phi_{n}(x)\right\}$ and $\left\{b_{n}\right\}$ as we did when proving the necessity in Theorem 3. Then the series

$$
\sum_{n=1}^{\infty} b_{n} \Phi_{n}(x)
$$

will not be $(c, 1)$-summable anywhere.

Remark 4. If

$$
N_{k}=\left[2^{2^{k^{\alpha}}}\right], \quad \alpha>\frac{1}{2}
$$

then the above-mentioned Kacmarz theorem will hold for all $\Delta_{k}$-ONS $\left\{\varphi_{n}(x)\right\}$. 
Theorem 5. Let the sequence $\left\{N_{k}\right\}$ be fixed. In order that the condition

$$
\sum_{n=1}^{\infty} a_{n}^{2} \omega(n)<\infty
$$

guarantee the convergence almost everywhere of the subsequence of partial sums $\left\{S_{2^{k}}(x)\right\}$ of series (1) with respect to any $\Delta_{k}-O N S\left\{\varphi_{n}(x)\right\}$, it is necessary and sufficient that the following two conditions be fulfilled:

(a) $\sum_{k=1}^{\infty} \frac{1}{\omega\left(N_{k}\right)}<\infty$;

(b) $\log _{2}^{2} k=O\left(\omega\left(M_{k}\right)\right)$ for $k \rightarrow \infty$, (13) where the sequence $\left\{M_{k}\right\}$ is defined by the recurrent formula (7).

Proof. Sufficiency. Let conditions (11), (12), (13) be fulfilled. Construct the system $\left\{\psi_{n}(x)\right\}$ and the sequence $\left\{b_{n}\right\}$ as we did when proving the sufficiency in Theorem 3. Set

$$
v(k)=\omega\left(M_{k}\right), \quad k \geq 1 .
$$

Then we obtain

$$
\begin{aligned}
\sum_{k=1}^{\infty} b_{k}^{2} v(k) & =\sum_{k=1}^{\infty}\left(\sum_{j=M_{k}+1}^{M_{k+1}} a_{j}^{2}\right) v(k)=\sum_{k=1}^{\infty}\left(\sum_{j=M_{k}+1}^{M_{k+1}} a_{j}^{2}\right) \omega\left(M_{k}\right) \leq \\
& \leq \sum_{k=1}^{\infty} \sum_{j=M_{k}+1}^{M_{k+1}} a_{j}^{2} \omega(j)<\infty, \\
\sum_{i=1}^{\infty} \frac{1}{v\left(k_{i}\right)} & =\sum_{i=1}^{\infty} \frac{1}{\omega\left(M_{k_{i}}\right)}=\sum_{i=1}^{\infty} \frac{1}{\omega\left(N_{i}\right)}<\infty .
\end{aligned}
$$

By condition (b) of Theorem 5 we have

$$
\log _{2}^{2} k=O\left(\omega\left(M_{k}\right)\right)=O(v(k)) \text { for } k \rightarrow \infty .
$$

Hence we conclude that $\left\{\psi_{n}(x)\right\}$ is an $\left(k_{i}, k_{i+1}\right]$-ONS and

$$
\sum_{i=1}^{\infty} \frac{1}{v\left(k_{i}\right)}<\infty, \quad \sum_{k=1}^{\infty} b_{k}^{2} v(k)<\infty, \quad \log _{2}^{2} k=O(v(k)) \text { for } k \rightarrow \infty .
$$

Now by Theorem 1 the series

$$
\sum_{n=1}^{\infty} b_{n} \psi_{n}(x)
$$

converges a.e. and therefore, in particular, it follows that the subsequence of partial sums $\left\{S_{2^{k}}(x)\right\}$ of the corresponding series (1) converges a.e.

Necessity. 
(1) Let

$$
\sum_{k=1}^{\infty} \frac{1}{\omega\left(N_{k}\right)}=\infty
$$

Construct $\left\{\Phi_{n}(x)\right\}$ and $\left\{b_{n}\right\}$ as we did in proving the necessity of condition (a) of Theorem 1. Then the sequence $\left\{S_{2^{k}}(x)\right\}$ diverges a.e. for series (1).

(2) Let

$$
\sum_{k=1}^{\infty} \frac{1}{\omega\left(N_{k}\right)}<\infty
$$

but

$$
\log _{2}^{2} k=c_{k} \omega\left(M_{k}\right), \quad k \geq 1
$$

where

$$
\varlimsup_{k \rightarrow \infty} c_{k}=\infty
$$

Let $v(k)=\omega\left(M_{k}\right)$. Then

$$
\log _{2}^{2} k=c_{k} v(k) \text { and } \varlimsup_{k \rightarrow \infty} c_{k}=\infty .
$$

Therefore there exist a $\left\{\Phi_{n}(x)\right\}$-ONS and a sequence $\left\{b_{k}\right\}$ (see Remark 1) such that

$$
\sum_{k=1}^{\infty} b_{k}^{2} v(k)<\infty
$$

but the series

$$
\sum_{k=1}^{\infty} b_{k} \Phi_{k}(x)
$$

diverges a.e.

Construct the system $\left\{\psi_{n}(x)\right\}$ and the sequence $\left\{a_{n}\right\}$. Namely, let

$$
a_{M_{i}}=b_{i}, \quad \psi_{M_{i}}(x)=\Phi_{i}(x), \quad i=1,2, \ldots .
$$

For the rest of $n \in\left(N_{i}, N_{i+1}\right]$ assume that $a_{n}=0$ and as $\psi_{n}(x)$ take anyone of the functions $\Phi_{k}(x), k \notin\left(k_{i}, k_{i+1}\right]$, so that $\psi_{i}(x) \neq \psi_{j}(x)$ for $i \neq j$ and $i, j \in \Delta_{k}$. In that case we obtain an $\Delta_{k}$-ONS $\left\{\psi_{n}(x)\right\}$ for which

$$
\sum_{n=1}^{\infty} a_{n}^{2} \omega(n)=\sum_{i=1}^{\infty} a_{M_{i}}^{2} \omega\left(M_{i}\right)=\sum_{i=1}^{\infty} b_{i}^{2} v(i)<\infty
$$

but the series

$$
\sum_{n=1}^{\infty} a_{n} \psi_{n}(x)
$$


diverges a.e. Then, following the construction of the terms of this series, the subsequence of partial sums $\left\{S_{M_{k}}(x)\right\}$, where $\left\{M_{k}\right\}$ is defined by (7), diverges a.e. But since

$$
\sum_{k=1}^{\infty} \frac{1}{\omega\left(N_{k}\right)}<\infty
$$

the subsequence of partial sums $\left\{S_{N_{k}}(x)\right\}$ of the series

$$
\sum_{n=1}^{\infty} a_{n} \psi_{n}(x)
$$

converges alsmost everywhere. Let the $\left\{S_{2^{n}}(x)\right\}$ converge on a set $E \subset$ $(0,1), m(E)>0$.

It is clear that from the sequences $\left\{N_{m}\right\}$ and $\left\{2^{n}\right\}$ we must obtain subsequences $\left\{N_{m_{k}}\right\}$ and $\left\{2^{n_{k}}\right\}$ such that

$$
S_{2^{n_{k}}}(x)-S_{N_{m_{k}}}(x)=a_{2^{n_{k}}} \psi_{2^{n_{k}}}(x), \quad k \geq 1 .
$$

Then

$$
\sum_{k=1}^{\infty} \int_{0}^{1}\left(S_{2^{n_{k}}}(x)-S_{N_{m_{k}}}(x)\right)^{2} d x \leq \sum_{k=1}^{\infty} a_{2^{n_{k}}}^{2}<\infty
$$

and therefore

$$
\lim _{k \rightarrow \infty}\left(S_{2^{n_{k}}}(x)-S_{N_{m_{k}}}(x)\right)=0 \quad \text { a.e. }
$$

i.e.,

$$
\begin{gathered}
\lim _{n \rightarrow \infty} S_{2^{n}}(x)=\lim _{k \rightarrow \infty} S_{2^{n_{k}}}(x)=\lim _{k \rightarrow \infty} S_{N_{m_{k}}}(x)=\lim _{m \rightarrow \infty} S_{N_{m}}(x) \\
\text { almost every } \quad x \in E,
\end{gathered}
$$

which contradicts the divergence a.e. of the sequence $\left\{S_{N_{k}}(x)\right\}$.

Theorem 6. Let the sequence $\left\{N_{k}\right\}$ be given and the equality

$$
\sum_{k=n}^{\infty} \frac{1}{N_{k}^{2}}=O\left(\frac{n}{N_{n}^{2}}\right) \text { for } n \rightarrow \infty
$$

be fulfilled.

In order that the positive nondecreasing sequence $\{\omega(n)\}$ be the Weyl multiplier for the $(c, 1)$-summability a.e. of series with respect to any $\Delta_{k}$ ONS, it is necessary and sufficient that conditions (12), and (13) be fulfilled.

Proof. Let condition (14) be fulfilled.

Sufficiency. Let conditions (11), (12) and (13) be fulfilled. Then for sufficiently large $k$ 's we have

$$
k<\omega\left(N_{k}\right)
$$


and therefore for sufficiently large $n$ 's

$$
\begin{gathered}
\min \left\{k: N_{k} \geq n\right\}+n^{2} \sum_{k: N_{k} \geq n} \frac{1}{N_{k}^{2}}=k(n)+1+n^{2} \sum_{k=k(n)+1}^{\infty} \frac{1}{N_{k}^{2}} \leq \\
\leq 2 k(n)+n^{2} \frac{c \cdot k(n)}{N_{k(n)+1}^{2}} \leq c k(n) \leq c \omega\left(N_{k(n)}\right)<c \omega(n)
\end{gathered}
$$

which yields

$$
\min \left\{k: N_{k} \geq n\right\}+n^{2} \sum_{k: N_{k} \geq n} \frac{1}{N_{k}^{2}}=O(\omega(n)) \text { for } n \rightarrow \infty .
$$

Then by Theorem 5 the sequence $\left\{S_{2^{k}}(x)\right\}$ converges a.e. for series (1), while by Theorem 2 series $(1)$ is $(c, 1)$-summable slmost everywhere.

Necessity.

(a) Let

$$
\sum_{k=1}^{\infty} \frac{1}{\omega\left(N_{k}\right)}=\infty
$$

Construct $\left\{\Phi_{n}(x)\right\}$ and $\left\{b_{n}\right\}$ as we did when proving the necessity of condition (a) of Theorem 1. Then we have

$$
\sum_{n=1}^{\infty} b_{n}^{2} \omega(n)<\infty
$$

and

$$
\sum_{n=1}^{\infty} b_{n} \Phi_{n}(x)=\sum_{k=1}^{\infty} b_{N_{k}}=\infty, \quad x \in(0,1),
$$

which imply that the series

$$
\sum_{n=1}^{\infty} b_{n} \Phi_{n}(x)
$$

is nowhere $(c, 1)$-summable.

(b) Let

$$
\sum_{k=1}^{\infty} \frac{1}{\omega\left(N_{k}\right)}<\infty
$$

but condition (13) be not fulfilled. Then by Theorem 5 there exist a $\Delta_{k^{-}}$ ONS $\left\{\psi_{n}(x)\right\}$ and a sequence $\left\{a_{n}\right\}$ such that

$$
\sum_{n=1}^{\infty} a_{n}^{2} \omega(n)<\infty
$$


but the corresponding subsequence of partial sums $\left\{S_{2^{k}}(x)\right\}$ diverges a.e. Moreover, if equality (15) is fulfilled, then by Theorem 2 the series

$$
\sum_{n=1}^{\infty} a_{n} \psi_{n}(x)
$$

is not $(c, 1)$-summable almost everywhere.

Remark 5. From the proof of Theorem 6 it is clear that condition (14) in this theorem can be replaced by condition (15). Then, assuming that $\omega(n)=\left(\log _{2} \log _{2} n\right)^{2}$ and condition (12) is fulfilled, by inequality (10) we have

$$
\log _{2}^{2} k=O\left(\left(\log _{2} \log _{2} M_{k}\right)^{2}\right) \text { for } k \rightarrow \infty,
$$

and by Lemma 3

$$
\min \left\{k: N_{k} \geq n\right\}+n^{2} \sum_{k: N_{k} \geq n} \frac{1}{N_{k}^{2}}=O\left(\left(\log _{2} \log _{2} n\right)^{2}\right) \text { for } n \rightarrow \infty,
$$

and we obtain Theorem 4 as a corollary.

Remark 6 . Theorem 6 implies that in the typical cases given below the Weyl multipliers for the $(c, 1)$-summability a.e. of series with respect to any $\Delta_{k}$-ONS are:

(a) if

$$
N_{k}=\left[2^{2^{k^{\alpha}}}\right], \quad 0<\alpha \leq \frac{1}{2},
$$

then

$$
\omega(n)=\left(\log _{2} \log _{2} n\right)^{\frac{1}{\alpha}+\varepsilon}, \quad \varepsilon>0 ;
$$

(b) if

$$
N_{k}=\left[2^{k^{\alpha}}\right], \quad \alpha>0,
$$

then

$$
\omega(n)=\left(\log _{2} n\right)^{\frac{1}{\alpha}+\varepsilon}, \quad \varepsilon>0
$$

(c) if

$$
N_{k}=\left[k^{\alpha}\right], \quad \alpha \geq 1,
$$

then

$$
\omega(n)=n^{\frac{1}{\alpha}}\left(\log _{2} n\right)^{1+\varepsilon}, \quad \varepsilon>0 .
$$

Note that if $\varepsilon=0$, then in cases (a), (b) and (c) $\{\omega(n)\}$ will be the Weyl multiplier not for each $\Delta_{k}$-ONS.

Remark 7. Condition (14) is fulfulled, in particular, if

$$
N_{k}=k \Phi(k),
$$

where $\Phi(k)$ does not decrease. 


\section{REFERENCES}

1. V. F. Gaposhkin, On the series by block-orthogonal and block-independent systems. (Russian) Izv. Vyssh. Uchebn. Zaved. Mat. 5(1990), $12-18$.

2. G. G. Nadibaidze, On some problems connected with series with respect to $\Delta_{k}$-ONS. Bull. Acad. Sci. Georgia 143(1991), No. 1, 16-19.

3. G. G. Nadibaidze, On some problems connected with series with respect to $\Delta_{k}$-ONS. Bull. Acad. Sci. Georgia 144(1991), No. 2, 233-236.

4. B. S. Kashin and A. A. Saakyan, Orthogonal series. (Russian) Nauka, Moscow, 1984;Engl. transl.: Translations of Mathematical Monographs, 75, Amer. Math. Soc., Providnce, RI, 1989.

5. S. Kaczmarz and H. Steinhaus, Theorie der Orthogonalreihen. Warszawa-Lwow, 1935.

(Received 16.02.1994)

Author's address:

Faculty of Mechanics and Mathematics

I. Javakhishvili Tbilisi State University

2, University St., Tbilisi 380043

Republic of Georgia 